\title{
BISECTRICES OF A TRIANGLE IN A LINEAR NORMED SPACE AND HILBERTIAN NORMS
}

BY

\section{IULIANA IONICĂ}

\begin{abstract}
In this paper we study some geometrical properties using the norm derivatives. We define the bisectrice of an angle and establish some characterizations of hilbertian norms in a smooth space.

Mathematics Subject Classification 2000: 46B, 46C15, 46C50.

Key words: smooth space, hilbertian norm, heights of a triangle, bisectrix of a triangle, incenter, Birkhoff orthogonality, isosceles orthogonality.
\end{abstract}

\section{Introduction}

Let $(X,\|\cdot\|)$ be a real linear normed space and let us denote the directional derivatives of the norm by:

$$
n_{ \pm}^{\prime}(x ; y)=\lim _{t \rightarrow \pm 0} \frac{\|x+t y\|^{2}-\|x\|^{2}}{2 t} \text {, for all } x, y \in X,
$$

limits which always exist $([3],[10])$. The basic properties of $n_{ \pm}^{\prime}$ to be frequently used are the following:

(i) $n_{ \pm}^{\prime}(x ; x)=\|x\|^{2}$ and $n_{ \pm}^{\prime}(x ; y) \leq\|x\| \cdot\|y\|, \forall x, y \in X$;

(ii) $n_{-}^{\prime}(x ; y) \leq n_{+}^{\prime}(x ; y), \forall x, y \in X$;

(iii) $n_{+}^{\prime}(-x ; y)=n_{+}^{\prime}(x ;-y)=-n_{-}^{\prime}(x ; y), \forall x, y \in X$;

(iv) $n_{+}^{\prime}(\alpha x ; \beta y)=n_{+}^{\prime}(\beta x ; \alpha y)=\alpha \beta n_{+}^{\prime}(x ; y), \forall x, y \in X$ and $\alpha, \beta \geq 0$.

(v) $n_{+}^{\prime}\left(x ; y_{1}+y_{2}\right) \leq n_{+}^{\prime}\left(x ; y_{1}\right)+n_{+}^{\prime}\left(x ; y_{2}\right), \forall x, y_{1}, y_{2} \in X$; 
(vi) $n_{+}^{\prime}(x ; \alpha x+y)=n_{+}^{\prime}(x ; y)+\alpha\|x\|^{2}, \forall x, y \in X$ and $\alpha \geq 0$.

If $n_{-}^{\prime}(x ; y)=n_{+}^{\prime}(x ; y)$, for all $x, y \in X$, then we say that the norm is Gâteaux differentiable, because $n_{+}^{\prime}(x, \bullet)$ is a linear continuous functional for each element $x \in X$. In this paper, whenever we will say that the norm is differentiable, we must understand the Gâteaux differentiability.

In a linear normed space, if the norm is differentiable, then we say that the space is smooth. For instance, a linear normed space is smooth whenever his dual is strictly convex ([3], [10]).

In this paper we study the problem of bisectrices of a triangle with the respect of Birkhoff orthogonality and we will also study some characterizations of linear normed spaces with the norm generated by an inner product using some metric properties of the heights and bisectrices of a triangle in a linear normed space, properties which are well-known in the Euclidian geometry.

Most of the properties which use the directional derivatives lead us frequently to the symmetry of directional derivatives, which allow us to use the next well-known characterization:

Theorem 1.1. Let $(X,\|\cdot\|)$ be a real linear normed space, $\operatorname{dim} X \geq 2$. Then $X$ is an inner product space if and only if for all linearly independent vectors $x, y$ in $X$

$$
n_{+}^{\prime}(x ; y)=n_{+}^{\prime}(y ; x)
$$

According to (iv) it is sufficient that the property (2) to be satisfied only in the points $x, y \in X$ with $\|x\|=\|y\|=1$.

If the space is smooth, this property has been established for the first time by LEDUC in [12]. And if $\operatorname{dim} X \geq 3$ it is sufficient the next property to be satisfied:

$$
n^{\prime}(x, y)=0 \Rightarrow n^{\prime}(y, x)=0
$$

(see [12], Theorem 3).

The non-smooth case has been established by AMIR in [2].

In [14], PAPINI shows that the property (2) it is sufficiently to be satisfied in the next weaker expression, i.e. we have:

$$
\left|n_{+}^{\prime}(x ; y)\right|=\left|n_{+}^{\prime}(y ; x)\right|, \text { for all } x, y \in X \text { with }\|x\|=\|y\|=1
$$


(see also [2, (2.5) pg. 18]).

In [16] we proved that the symmetry property (2) is sufficiently to be satisfied only in the pairs of points in which the norm is quite differentiable, taking into account that for an arbitrary norm, the set of differentiable points is dense in the space $([3],[9])$. In fact, most of the characterizations of hilbertian norms established by different authors in the case of smooth spaces can be easily extended asking for those properties to be satisfied only in the points where the norm is quite differentiable (see, for instance [15]).

\section{Orthogonality relations}

Let $(X,\|\cdot\|)$ be a general real linear normed space, endowed with the following orthogonality relation:

$$
x \perp y \Leftrightarrow\|x+t y\| \leq\|x\|, \quad \text { for all } x, y \in X \text { and } t \in \mathbb{R},
$$

which usually it is known as Birkhoff orthogonality ([5]).

Also, on $X$ we can define another important orthogonality relation, namely:

$$
x \neq y \Leftrightarrow\|x+y\|=\|x-y\|, \text { for all } x, y \in X,
$$

which usually it is known as isosceles orthogonality.

The basic properties of this types of othogonality and the connections with other types of orthogonalities in a linear normed space were established in $[2],[6],[7],[11]$.

Taking into account the property of minimum elements of a convex function (see, for example, [3], [9], [10]), if the space is smooth, this type of orthogonality can be characterized using the differential of the norm. Precisely:

$$
x \perp y \Leftrightarrow n^{\prime}(x, y)=0 .
$$

In the case of the norms which are not necessarily Gateaux differentiable, the orthogonality condition can be characterized by directional derivatives of the norm:

$$
x \perp y \Leftrightarrow n_{-}^{\prime}(x ; y) \leq 0 \leq n_{+}^{\prime}(x ; y) .
$$

Let $(X,\|\cdot\|)$ be a real smooth linear normed space, $\operatorname{dim} X \geq 2, x, y \in X$ two linearly independent vectors and the triangle of vertices $0, x, y$. 
Using the orthogonality properties, in [16] we defined the height vector by:

$$
h(x ; y)=y+\frac{n^{\prime}(y-x ; y)}{\|x-y\|^{2}}(x-y), \quad \text { for all } x, y \in X .
$$

Indeed, the following orthogonality property holds: $(x-y) \perp h(x ; y)$, for all $x, y \in X$. In [1], Alsina, Guijarro and Tomas obtained another expression for the height vector, namely:

$$
h(x ; y)=y+\frac{\|y\|^{2}-n_{+}^{\prime}(x ; y)}{\|x-y\|^{2}}(x-y), \text { for all } x, y \in X .
$$

In the case of smooth linear normed spaces it can be easily verified that the heights given by (8) and (9) are the same for any triangle if and only if the norm of the space is hilbertian (see [16]).

\section{Bisectrix in a triangle}

Let us suppose for the beginning that $X$ is a smooth linear normed space endowed with Birkhoff orthogonality, which in this spaces can be equivalently defined by (7).

So, let us consider $x, y \in X$ linearly independent and for simplicity we can consider the triangle with vertices $0, x, y$. Using (7) we get:

$$
\begin{aligned}
& n^{\prime}(\alpha y ; t x+(1-t) y-\alpha y)=0, \\
& n^{\prime}(\beta x ; t x+(1-t) y-\beta x)=0, \quad \text { where } \alpha, \beta \in \mathbb{R} .
\end{aligned}
$$

where $b(x, y)=t x+(1-t) y$ is a point of bisectrix.

Now, let us define the bisectrix of the angle $x 0 y$ by the Euclidian condition:

$$
\|t x+(1-t) y-\alpha y\|=\|t x+(1-t) y-\beta x\|,
$$

which has the unique solution

$$
t=\frac{\|y\|^{2} \cdot\|\| x\left\|^{2} y-n^{\prime}(x ; y) x\right\|}{\|\| y\left\|^{2} x-n^{\prime}(y ; x) y\right\|\|\|\|x\|^{2} y-n^{\prime}(x ; y) x\|\cdot\| y \|^{2}} .
$$


Thus, from (10) and (11) we obtain:

$$
\begin{aligned}
b(x, y) & =\frac{\|y\|^{2} \cdot\|\| x\left\|^{2} y-n^{\prime}(x ; y) x\right\|}{\|\| y\left\|^{2} x-n^{\prime}(y ; x) y\right\| \cdot\|x\|^{2}+\|\| x\left\|^{2} y-n^{\prime}(x ; y) x\right\| \cdot\|y\|^{2}} x+ \\
& +\frac{\|x\|^{2} \cdot\|\| y\left\|^{2} x-n^{\prime}(y ; x) y\right\|}{\|\| y\left\|^{2} x-n^{\prime}(y ; x) y\right\| \cdot\|x\|^{2}+\|\| x\left\|^{2} y-n^{\prime}(x ; y) x\right\| \cdot\|y\|^{2}} y .
\end{aligned}
$$

And if we denote by

$$
k(x ; y)=\frac{\|y\|^{2} \cdot\|\| x\left\|^{2} y-n^{\prime}(x ; y) x\right\|}{\|x\|^{2} \cdot\|\| y\left\|^{2} x-n^{\prime}(y ; x) y\right\|},
$$

then $k(y ; x)=\frac{1}{k(x ; y)}$ and $k(x ; y)=k(-x ; y)=k(x ;-y)$.

Therefore, we get the next form for the bisectrices in any triangle having a vertex in the origin:

$$
\begin{aligned}
& b_{0 ; x, y}: \alpha\left[\frac{1}{1+k(y ; x)} x+\frac{1}{1+k(x ; y)} y\right], \quad \alpha \in \mathbb{R}, \\
& b_{x ; y, 0}: \alpha \frac{1}{1+k(x ; y-x)} \cdot y+(1-\alpha) \cdot x, \quad \alpha \in \mathbb{R}, \\
& b_{y ; 0, x}: \alpha \frac{1}{1+k(y ; y-x)} x+(1-\alpha) \cdot y, \quad \alpha \in \mathbb{R} .
\end{aligned}
$$

Because $k(y ; x)=\frac{1}{k(x ; y)}$, we observe that we also have:

$$
b_{0 ; x, y}: \alpha \cdot[k(x ; y) x+y], \quad \alpha \in \mathbb{R} .
$$

And if we replace $x$ by $\frac{x}{\|x\|}$ and $y$ by $\frac{y}{\|y\|}$ we get:

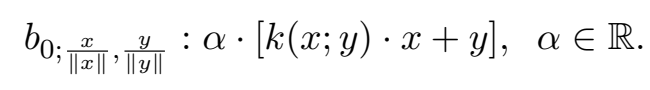

In spaces with the norm not necessarily differentiable, the bisectrix can be given using the directional derivatives and the property $\left(7^{\prime}\right)$. For example, if the norm is not differentiable the bisectrix from the origin can be considered as:

$$
\begin{aligned}
& b_{0 ; x, y}: \alpha\left[\frac{\|y\|^{2} \cdot\|\| x\left\|^{2} y-a(x ; y) x\right\|}{\|\| y\left\|^{2} x-a(y ; x) y\right\| \cdot\|x\|^{2}+\|\| x\left\|^{2} y-a(x ; y) x\right\| \cdot\|y\|^{2}} x\right. \\
&\left.\quad+\frac{\|x\|^{2} \cdot\|\| y\left\|^{2} x-a(y ; x) y\right\|}{\|\| y\left\|^{2} x-a(y ; x) y\right\| \cdot\|x\|^{2}+\|\| x\left\|^{2} y-a(x ; y) x\right\| \cdot\|y\|^{2} \cdot y}\right]
\end{aligned}
$$


where $\alpha \in \mathbb{R}$ and $a(x ; y) \in \mathbb{R}$ has the following property:

$$
n_{-}^{\prime}(x ; y) \leq a(x ; y) \leq n_{+}^{\prime}(x ; y) .
$$

If $X$ is a space with a semi-inner product in Lumer's sense ([8], [13]), it is natural that the bisectrix to be considered:

$$
\begin{aligned}
b_{0, x ; y} & : \alpha\left[\frac{\|y\|^{2} \cdot\|\| x\left\|^{2} y-[x ; y] x\right\|}{\|\| y\left\|^{2} x-[y ; x] y\right\| \cdot\|x\|^{2}+\|\| x\left\|^{2} y-[x ; y] x\right\| \cdot\|y\|^{2}} x\right. \\
& \left.+\frac{\|x\|^{2} \cdot\|\| y\left\|^{2} x-[y ; x] y\right\|}{\|\| y\left\|^{2} x-[y ; x] y\right\| \cdot\|x\|^{2}+\|\| x\left\|^{2} y-[x ; y] x\right\| \cdot\|y\|^{2}} y\right]
\end{aligned}
$$

where $\alpha \in \mathbb{R}$ and $[.,$.$] is the semi-inner product in X$.

If the norm is differentiable on $X$, it is well-known that $[x, y]=n^{\prime}(x, y)$ for all $x, y \in X$.

Now, taking into account the action in case of translation of properties (10) and (11), we can define the bisectrices of any triangle with vertices $x, y, z \in X$, which are not all on the same straight as it follows:

$$
\begin{aligned}
& b_{x ; y, z}: x+\alpha \cdot b(y-x ; z-x) \\
& b_{y ; z, x}: y+\alpha \cdot b(z-y ; x-y), \quad \alpha \in \mathbb{R}, \\
& b_{z ; x, y}: z+\alpha \cdot b(x-z ; y-z) .
\end{aligned}
$$

Using (14), we get:

(20) $b_{x ; y, z}: \alpha\left[\frac{1}{1+k(y-x, z-x)} \cdot z+\frac{1}{1+k(z-x, y-x)} y\right]+(1-\alpha) x$,

where $\alpha \in \mathbb{R}$ and the vertices $x, y, z \in X$ are three arbitrary elements which are not all on the same straight.

Remark 3.1. Taking into account the manner we determined the bisectices in a triangle, it is natural to say that all three bisectrices of a triangle are meeting in an unique point, called the incenter of the triangle.

\section{Some characterization of hilbertian norms}

In order to give some characterizations of hilbertian norms, we will use some results in the theory of semi-inner products in a strictly convex space. 
Definition 4.1. A real linear normed space, $X(\operatorname{dim} X \geq 2)$ is called strictly convex if whenever $\|x\|+\|y\|=\|x+y\|$, where $x, y \neq 0$, then $y=\lambda x$, for some real $\lambda>0$.

It is convenient to characterize strict convexity of the norm in terms of semi-inner product properties. In this way BeRKSON [4, p. 281], proved the next property:

Lemma 4.2. A semi-inner product space is strictly convex if and only if whenever $[x, y]=\|x\| \cdot\|y\|$, where $x, y \neq 0$, then $y=\lambda x$, for some real $l>0$.

Lemma 4.3. Let $X$ be a smooth linear normed space, strictly convex, $\operatorname{dim} X \geq 3$. Then $X$ is an inner-product space if and only if for all linearly independent vectors $x, y \in X$ in we have:

$$
\|x\| \cdot\|\| y\left\|^{2} x-n^{\prime}(y ; x) y\right\|=\|y\| \cdot\|\| x\left\|^{2} y-n^{\prime}(x ; y) x\right\| .
$$

Proof. If we assume that property (21) holds and if we suppose $n^{\prime}(x, y)=$ 0 , we obtain $\left\|n^{\prime}(y ; x) y-\right\| y\left\|^{2} x\right\|=\|x\| \cdot\|y\|^{2}$. But, $n^{\prime}\left(x ;\|y\|^{2} x-n^{\prime}(y ; x) y\right)=$ $\|x\|^{2}\|y\|^{2}-n^{\prime}(y ; x) \cdot n^{\prime}(x ; y)=\|x\|^{2}\|y\|^{2}$. So, we get:

$$
n^{\prime}\left(x ;\|y\|^{2} x-n^{\prime}(y ; x) y\right)=\|x\|\|\| y\left\|^{2} x-n^{\prime}(y ; x) y\right\| .
$$

Using Lemma 4.1, it follows that $\|y\|^{2} x-n^{\prime}(y ; x) y=t x$, for certain $t>0$. As $x, y$ are linearly independent in $X$, we have: $\|y\|^{2}=t$ and $n^{\prime}(y ; x)=0$. Shortly, we supposed that $n^{\prime}(x, y)=0$ and we obtained $n^{\prime}(y ; x)=0$. Since $\operatorname{dim} X \geq 3$, this means, according to property (3), that the norm on $X$ is hilbertian.

The next characterization recalls a well-known property in Euclidian geometry, namely the bisectrix theorem.

Theorem 4.4. Let $X$ be a smooth linear normed space, strictly convex, $\operatorname{dim} X \geq 3$. Then $X$ is an inner-product space if and only if for all linearly independent vectors $x, y$ in $X$, the next property holds:

$$
\frac{\|b(x ; y)-x\|}{\|b(x ; y)-y\|}=\frac{\|x\|}{\|y\|} .
$$


Proof. If $X$ is an inner-product space, we can immediately verify that the condition (22) is satisfied. Now, if we assume (22), using (14), we get:

$$
\frac{\left\|\left(\frac{1}{1+k(y ; x)}-1\right) x+\frac{1}{1+k(x ; y)} y\right\|}{\left\|\frac{1}{1+k(y ; x)} x+\left(\frac{1}{1+k(x ; y)}-1\right) y\right\|}=\frac{\|x\|}{\|y\|} .
$$

And after a simply computation, we obtain:

$$
\frac{\|\| y\left\|^{2} x-n^{\prime}(y ; x) y\right\|}{\|\| x\left\|^{2} y-n^{\prime}(x ; y) x\right\|}=\frac{\|y\|}{\|x\|}, \text { namely } \quad(21) .
$$

So, using Lemma 4.2., the proof is finished.

Remark 4.5. We know that in an inner product space, if we consider an isosceles triangle (meaning two sides with equal length), then the bisectrix vector divides the third side into two segments of equal length. This property characterizes the inner product spaces and is an immediately conclusion using the bisectrix theorem.

The next theorem gives us characterizations of hilbertian norms using some properties from the Euclidian geometry. We must say that it is sufficient to consider all this in the case of $\|x\|=\|y\|=1$, without any loss of generality.

Theorem 4.6. Let $X$ be a smooth linear normed space, strictly convex, $\operatorname{dim} X \geq 3$. Then the following conditions are equivalent:

(i) $X$ is an inner product space;

(ii) $\|x\| \cdot\|h(-y ; x-y)=\| y\|\cdot\| h(y-x ;-x \|)$, for all linearly independent $x, y \in X$ with $\|x\|=\|y\|=1$;

(iii) $\|x-y-b(x-y ;-y)\|=\| y-x-b(y-x ;-x)$, for all linearly independent $x, y \in X$ with $\|x\|=\|y\|=1$;

(iv) $\frac{n^{\prime}(x ; b(x ; y))}{\|x\|}=\frac{n^{\prime}(y ; b(x ; y))}{\|y\|}$, for all linearly independent $x, y \in X$ with $\|x\|=\|y\|=1$.

Proof. (i) $\rightarrow$ (ii) and (i) $\rightarrow$ (iii) are immediate. 
Firstly, if we assume (ii), using (8) we get:

$$
\|x\| \cdot\left\|x-y+\frac{n^{\prime}(x ; x-y)}{\|x\|^{2}}=\right\| y\|\cdot\|-x+\frac{n^{\prime}(y ; x)}{\|y\|^{2}}(-x) \| .
$$

After a simply computation, we obtain:

$$
\|x\| \cdot\|\| y\left\|^{2} x-n^{\prime}(y ; x) y\right\|=\|y\| \cdot\|\| x\left\|^{2} y-n^{\prime}(x ; y) x\right\|
$$

and using Lemma 4.2 it follows that $X$ is an inner product space. Now, let's assume the condition (iii) and using (14) we get:

$$
\begin{aligned}
& \left\|x-y-\frac{1}{1+k(-y ; x-y)}(x-y)-\frac{k(-y ; x-y)}{1+k(-y ; x-y)}(-y)\right\| \\
& =\left\|y-x-\frac{1}{1+k(-x ; y-x)}(y-x)-\frac{k(-x ; y-x)}{1+k(-x ; y-x)}(-x)\right\| .
\end{aligned}
$$

After a simply computation we obtain: $k(-y ; x-y)=k(-x ; y-x)$, which leads us to: \|\|$y\left\|^{2} x-n^{\prime}(y ; x) y\right\|=\|\| x\left\|^{2} y-n^{\prime}(x ; y) x\right\|$ and using again Lemma 4.2 it means the norm on $X$ is hilbertian. And now, if we assume that (iv) holds, then we get:

$$
\|x\| \cdot n^{\prime}(y ; x+k(y ; x) y)=\|y\| \cdot n^{\prime}(x ; x+k(y ; x) y) .
$$

Replacing $k(y ; x)$ in $(23)$, we obtain:

$$
\begin{aligned}
& \|\| y\left\|^{2} x-n^{\prime}(y ; x) y\right\| \cdot\left[n^{\prime}(x ; y)-\|x\| \cdot\|y\|\right] \\
& =\|\| x\left\|^{2} y-n^{\prime}(x ; y) x\right\| \cdot\left[n^{\prime}(y ; x)-\|x\| \cdot\|y\|\right] .
\end{aligned}
$$

If we substitute $y \rightarrow-y$, the next equality follows:

$$
\begin{aligned}
& \|\| y\left\|^{2} x-n^{\prime}(y ; x) y\right\| \cdot\left[n^{\prime}(x ; y)+\|x\| \cdot\|y\|\right] \\
& =\|\| x\left\|^{2} y-n^{\prime}(x ; y) x\right\| \cdot\left[n^{\prime}(y ; x)+\|x\| \cdot\|y\|\right] .
\end{aligned}
$$

If we divide (24) by (25), we obtain:

$$
\frac{n^{\prime}(x ; y)-\|x\| \cdot\|y\|}{n^{\prime}(x ; y)+\|x\| \cdot\|y\|}=\frac{n^{\prime}(y ; x)-\|x\| \cdot\|y\|}{n^{\prime}(y ; x)+\|x\| \cdot\|y\|} .
$$

After a simply computation we obtain: $n^{\prime}(x ; y)=n^{\prime}(y ; x)$ and so, using Theorem 1.1, we get that the norm is hilbertian. With all these, the proof is complete. 
In Euclidian geometry, it is well-known that the bisectrices of an angle (interior and exterior) are orthogonal. We will proof that in linear normed spaces this property characterizes the hilbertian norms, of course in certain conditions.

In this way, let us consider two linearly independent vectors $x, y$ in $X$ and the triangle of vertices $0, x, y$. Obviously, the exterior bisectrix of the angle from the origin is as follows:

$$
b_{0 ;-x, y}: \alpha\left[\frac{1}{1+k(y,-x)}(-x)+\frac{1}{1+k(-x, y)} y\right] \text {, where } \alpha \in \mathbb{R} .
$$

Theorem 4.7. If $X$ is strictly convex, with $\operatorname{dim} X \geq 3$, then the norm on $X$ is hilbertian if and only if the interior and exterior bisectrices of the angle determined by $x$ and $y$ are orthogonal in isosceles' sense, whenever $x, y$ are two linearly independent vectors in $X$.

Proof. Indeed, if $X$ is an inner-product space, the property is immediately. Let us consider now that $b(x ; y) \neq b(-x ; y)$. This means:

$$
\|b(x ; y)+b(-x ; y)\|=\|b(x ; y)-b(-x ; y)\| .
$$

After a simply computation we obtain: $k(y, x)=\frac{\|y\|}{\|x\|}$, meaning $\|x\| \cdot\|y\|^{2} x-$ $n^{\prime}(y ; x) y\|=\| y\|\cdot\|\|x\|^{2} y-n^{\prime}(x ; y) x \|$ and using Lemma 4.2 we obtain that the norm is hilbertian.

There are other ways to define the bisectrices of a triangle. For instance, in [1], Alsina, GuiJarro and Tomas have defined the bisectrix replacing the metric property (10) with the property of equality between angles. In this case the bisectrix of an angle determined by two linearly independent vectors $x, y$ in $X$ is:

$$
b(x ; y)=\frac{\|y\|}{\|y\|+\|x\| k(y ; x)} x+\frac{\|x\|}{\|x\|+\|y\| k(x ; y)} y,
$$

where

$$
k(x ; y)=\frac{\|x\| \cdot\|y\|-n^{\prime}(x ; y)}{\|x\| \cdot\|y\|-n^{\prime}(y ; x)} .
$$

According to Theorem 1.1, it is obvious that the bisectrices given by (13) and (31) are the same if and only if the norm of the space is hilbertian. On the other side, in any triangle the three bisectrices given by (31) are not meeting any more in an unique point. If we will ask for the bisectrices to 
meet in an unique point, we will obtain again a characterization of hilbertian norms (see [17]).

\section{REFERENCES}

1. Alsina, C.; Guijarro, P.; TomŚs, M.S. - Some remarkable lines of triangles in real normed spaces and characterizations of inner product structures, Aequationes Math., 54 (1997), 234-241.

2. Amir, D. - Characterizations of Inner Product Spaces, Séminaire d'Analyse Fonctionelle 1984/1985, 77-93, Publ. Math. Univ. Paris VII, 26, Univ. Paris VII, Paris, 1986.

3. Barbu, V.; Precupanu, Th. - Convexity and Optimization in Banach Spaces, Mathematics and its Applications (East European Series), 10, D. Reidel Publishing Co., Dordrecht, 1986.

4. Berkson, E. - Some types of Banach spaces, Hermitian operators, and Bade functionals, Trans. Amer. Math. Soc., 116 (1965), 376-385.

5. Birkhoff, G. - Orthogonality in linear metric spaces, Duke Math. J., 1 (1935), 169-172.

6. Day, M.M. - Some characterizations of inner-product spaces, Trans. Amer. Math. Soc., 62 (1947), 320-337.

7. Day, M.M. - Normed Linear Spaces, Third edition, Ergebnisse der Mathematik und ihrer Grenzgebiete, Band 21, Springer-Verlag, New York-Heidelberg, 1973.

8. Giles, J.R. - Classes of semi-inner-product spaces, Trans. Amer. Math. Soc., 129 (1967), 436-446.

9. Giles, J.R. - Convex Analysis with Application in the Differentiation of Convex Functions, Research Notes in Mathematics, 58, Pitman (Advanced Publishing Program), Boston, Mass.-London, 1982.

10. Holmes, R.B.-Geometric Functional Analysis and its Applications, Graduate Texts in Mathematics, 24, Springer-Verlag, New York-Heidelberg, 1975.

11. James, R.C. - Orthogonality in normed linear spaces, Duke Math. J., 12 (1945), 291-302.

12. Leduc, M. - Caractérisation des espaces euclidiens, C.R. Acad. Sci. Paris Sér. A-B, 268 (1969), 943-946.

13. Lumer, G. - Semi-inner-product spaces, Trans. Amer. Math. Soc., 100 (1961), 29-43.

14. PAPINI, P.L. - Inner products and norm derivatives, J. Math. Anal. Appl., 91 (1983), $592-598$ 
15. Precupanu, T. - Characterizations of Hilbertian norms, Boll. Un. Mat. Ital. B, 15 (1978), 161-169.

16. Precupanu, T.; Ionică, I. - Heights of a triangle in a linear normed space and Hilbertian norms, An. Ştiinţ. Univ. "Al. I. Cuza" Iaşi. Mat. (N.S.), 55 (2009), 35-47.

17. Tomás, M.S. - Incenters in real normed spaces, Aequationes Math., 67 (2004), 63 72.

Received: 23.IV.2010

Department of Mathematics,

University "Al.I. Cuza", 11, Bd. Carol I, 700506, Iaşi, ROMANIA

ionica_iuliana@yahoo.com 